\title{
On-line Faults Signature Monitoring Tool for Induction Motor Diagnosis
}

\begin{abstract}
Ammar Medoued ${ }^{\dagger}$, Abdesselem Lebaroud*, Ahcene Boukadoum* and Guy Clerc**
Abstract - The monitoring and the diagnosis of the faults in induction motors starting from the stator current are very interesting, since it is an accessible and measurable quantity. The spectral analysis of the stator current makes it possible to highlight the characteristic frequencies of the faults but in a wide frequency range depending on half the sampling frequency, making it very difficult to monitor on-line the faults. In order to facilitate the use of the relevant frequencies of machine faults we proposed the extraction of the frequency components using two methods, namely, the amplitude and the instantaneous frequency. The theoretical bases of these methods were presented and the results were validated on a test bench with an induction motor of $5.5 \mathrm{kw}$.
\end{abstract}

Keywords: On-line Monitoring, Diagnosis, Induction Motor, Instantaneous amplitude, Instantaneous frequency

\section{Introduction}

Induction machines are increasingly used in electric drives. This machine, in the last three decades, has been the subject of several research works directed primarily towards the design of control laws ever more efficient. Its multiple qualities: low cost, robustness and performances make it a special familiar machine in industrial environment. The requirements of reliability and productivity of installations require the integration of a system of monitoring and diagnosis of failures. It must be necessary, therefore, to provide the motors with some tools for monitoring and diagnosis of faults, thus we will be able to avoid and prevent the breakdowns and the spurious shutdowns. The techniques and methods for monitoring and diagnosis treated in the literature [1]-[5] based on monitoring the temporal evolution and/or the spectral content of the signal. Most are based on direct analysis of the spectrum of the measured variable. They allow to extract a particular frequency (demodulation, filtering), a frequency band (filtering, FFT).

It is about methods known as MCSA (Motor Current Signature Analysis) methods. Signal processing, particularly the spectral analysis of vibration or electrical signals, is frequently used to detect faults in electric machines, in particular the fault of rotor breaking bars, stator faults and bearings faults. This method is particularly suited to this type of faults since these latter emerge by the appearance of certain frequencies, directly related to the speed of the machine and the frequency of the power supply. The methods of spectral analysis are used on electrical stationary

$\dagger$ Corresponding Author: Dept. of Electrical Engineering, Skikda University, Algeria.(lebaroud80@gmail.com)

* Dept. of Electrical Engineering, Skikda University, Algeria.

** Dept. of Electrical Engineering, Claude Bernard University, France. ( clerc@cegely.univ-lyon1.fr)

Received : September 12, 2009 ; Accepted : February 2, 2010 quantities when the electrical machine is connected to the network or to a frequency converter. The case of nonstationarity has also been used in the method of the disconnection of power to the motor [6], where the spectrum of the residual emf in the stator windings is used for the detection of rotor and stator faults. Monitoring of characteristic frequencies allow the characterization of many faults in the induction machine and this, sometimes independently of the amplitude of the measured signals (the occurrence or the modification of a characteristic frequency determines the presence of the fault and not the amplitude of the measured signal). On-line monitoring of faults through the spectral analysis is not an easy task because of the presence of all the frequency components included in the signal especially the fundamental that may sometimes inhibit the default characteristic components at low spectral resolution.

The techniques of the amplitude and instantaneous frequency can overcome these problems and allow a better extraction and exploitation of the frequency component specific to the failure.

\section{Detection of Faults by Instantaneous Amplitude}

\subsection{Single Phase Current}

The current in a stator phase of an electric motor can be developed in Fourier series:

$$
i_{a}(t)=\sum_{k} \sqrt{2} I_{a k} \sin \left(k \omega t+\varphi_{a k}\right)
$$

Note that $k_{M}$ represents the order of the harmonic with the maximum frequency after filtering of the current $i_{a}(t)$

$$
i_{a}(m)=\sum_{k=1}^{k_{M}} \sqrt{2} I_{a k} \sin \left(k \omega m \Delta t+\varphi_{a k}\right)
$$


Where $k_{M}$ is a real positive representing the range of harmonic components. $I_{a k}, k \omega, \varphi_{a k}$ are, Effective (rms), angular frequency and phase angle of the $k^{\text {th }}$ harmonic respectively. Each harmonic $i_{a}(t)$ can be represented by the harmonic phaser.

$$
I_{a k}=I_{k} \exp \left(j \varphi_{a k}\right)
$$

Given a real signal, in this case the stator phase current of the expression (3), the instantaneous amplitude is the module of the Hilbert transform of the considered signal. Since the signal is broadband and contains harmonics from various sources (source pollution or harmonics induced by the machine itself), we will remove the unwanted harmonics by filtering the current signal by a low pass filter with a cut off frequency of $100 \mathrm{~Hz}$. The principle of the instantaneous amplitude is illustrated in Fig. 1.

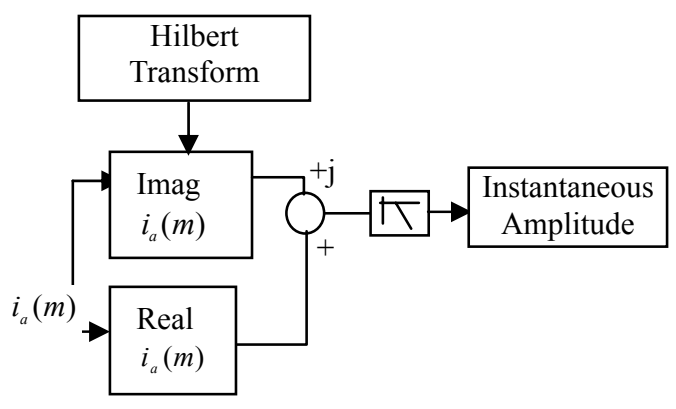

Fig. 1. Principle of the instantaneous amplitude.

\subsection{Three-phase Currents System}

The developed method is based on two transformations dealing with a projection of the three-phase quantities $i_{1}, i_{2}$ and $i_{3}$ in a fixed reference frame $\alpha, \beta$ and with the transformation from the fixed reference frame $(\alpha, \beta)$ towards a reference frame $(d, q)$ rotating in a reverse way at $-f_{\mathrm{e}}$ where $f_{\mathrm{e}}$ is the fundamental frequency.

The transformation of the three-phase currents system in the fixed reference frame leads to:

$$
\left[\begin{array}{c}
i_{\alpha} \\
i_{\beta}
\end{array}\right]=\frac{2}{3} \cdot\left[\begin{array}{ccc}
1 & -1 / 2 & -1 / 2 \\
0 & \sqrt{3} / 2 & -\sqrt{3} / 2
\end{array}\right] \cdot\left[\begin{array}{c}
i_{1} \\
i_{2} \\
i_{3}
\end{array}\right]
$$

The transformation of the current vector from the fixed reference $i_{\alpha \beta}$ towards the reverse rotating frame gives:

$$
\begin{gathered}
{\left[\begin{array}{l}
i_{d} \\
i_{q}
\end{array}\right]=\left[\begin{array}{ll}
\cos \left(\omega_{s} t\right) & -\sin \left(\omega_{s} t\right) \\
\sin \left(\omega_{s} t\right) & \cos \left(\omega_{s} t\right)
\end{array}\right] \cdot\left[\begin{array}{c}
i_{\alpha} \\
i_{\beta}
\end{array}\right]} \\
\bar{i}_{d q}=i_{d}+j i_{q}=\bar{I}_{-1}+\bar{I}_{1} e^{j 2 \omega_{s} t}+\sum_{h=1}^{n 1} \bar{I}_{6 h \pm 1} e^{ \pm j(6 h \pm 2) \omega_{s} t}
\end{gathered}
$$

The stator current vector (6) contains two alternate components and a direct one that corresponds to the negative sequence current. The transformation of a three-phase system of currents to a two phase system leads to a slip of all the frequencies of the same range. Consequently, the negative sequence current component at $\left(-f_{S}\right)$ frequency slips towards the DC.

\subsubsection{Analogical Demodulation}

In order to keep only the DC component, the analogical demodulation is performed on the low pass filtering of two components, one at twice the stator frequency and the other due to the harmonics. The summation of the squares of each term (Fig. 2) provides the amplitude of the negative sequence current component. The choice of the cut-off frequency is facilitated through the fact that current frequencies are multiple of the electric frequency. This approach offers a fast answer that solely depends on the transient response of the filter.

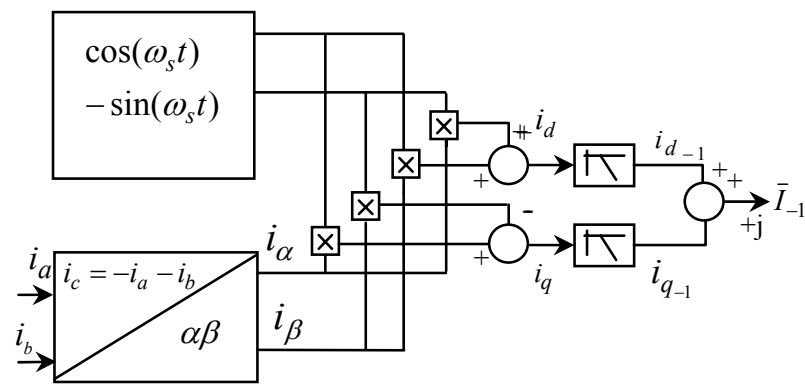

Fig. 2. Analogical demodulation of the stator currents vector.

\subsubsection{Numerical Demodulation}

The principle of the numerical demodulation (Fig. 3) is similar to that of the analogical one. The only difference lies in the numerical average of the summation of squares of each term of $i_{d}$ and $i_{q}$ which replace the low-pass filtering. Consequently, the harmonics alternate components (6) of the fundamental frequency are cancelled. This allows preserving the DC component only. In order to ensure their cancellation by the numerical demodulation, the sampling rate has to be selected greater than $400 \mathrm{~Hz}$ and as a multiple of the fundamental frequency.

The fault frequency $\left(f_{f}\right)$ of the negative sequence component of currents vector is $f_{f}=-f$ or $f_{f}=2 f=100 \mathrm{~Hz}$

$f=50 \mathrm{~Hz}$ : fundamental frequency

The minimum samples which allow calculation of the negative component are determinate with the following formula:

$$
N=\frac{f_{s}}{f_{f}}=\frac{f_{s}}{2 f}
$$

$f_{s}:$ sampling frequency 
Note also that the formula gives a zero mean value for all the alternative components of the stator current vector above $100 \mathrm{~Hz}$.

$N=4$ is the minimum number that allows obtain a mean of zero for the multiple components of $50 \mathrm{~Hz}$, so minimum sampling frequency is $f_{S}=N . f_{f}=400 \mathrm{~Hz}$

The computation of the negative sequence component of the current is carried out using the following expression:

$$
\bar{I}_{-1}=\frac{1}{N} \sum_{n=1}^{N} i_{d q}[n]
$$

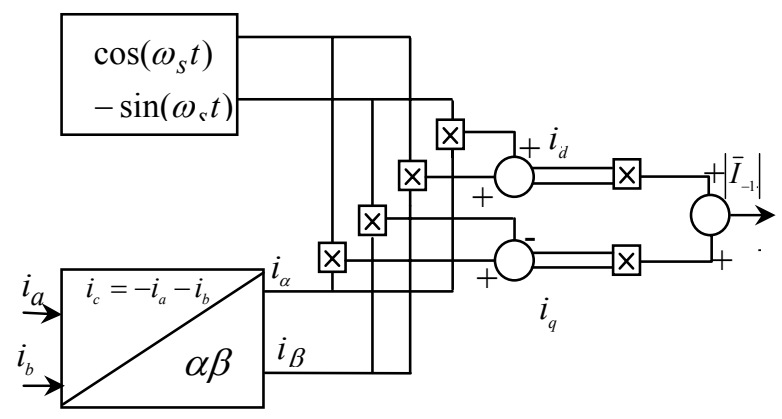

Fig. 3. Numerical demodulation of the stator currents vector.

\section{Detection of Faults by the Instantaneous Frequency}

\subsection{Principle}

The instantaneous frequency $f(t)$ uses the notion of the signal instantaneous phase, it is defined as the derivative of the phasej ( $t$ ) (Fig. 4).

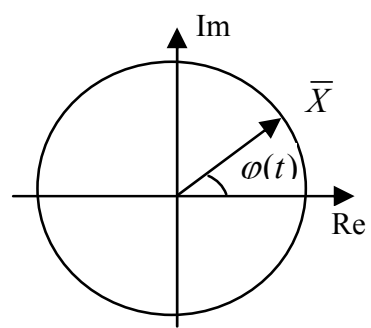

Fig. 4. Vector representation.

Consider a real signal $x(t)$, we obtain the phase $\mathrm{j}(t)$ from the associated signal $y(t)$ in quadrature. This latter is determined by the Hilbert transform:

$$
y(t)=\frac{1}{\pi} \int_{-\infty}^{+\infty} \frac{x(t)}{t-\tau} d \tau
$$

Which, from the real signal, provides the complex form:

$$
x(t)+j y(t)
$$

The phase $\mathrm{j}$ is the arctangent between $\mathrm{y}(\mathrm{t})$ et $\mathrm{x}(\mathrm{t})$.

$$
f_{i}=\frac{1}{2 \pi} \frac{d}{d t} \arctan \left(\frac{y}{x}\right)=\frac{1}{2 \pi} \frac{x y^{\prime}-y x^{\prime}}{x^{2}+y^{2}}
$$

Where: $x^{\prime}=d x / d t$ and $y^{\prime}=d y / d t$

$$
x^{\prime}=d x / d t \text { et } y^{\prime}=d y / d t
$$

The current instantaneous symmetrical component, allows us to directly have two quantities in quadrature of phase which are the real and imaginary components of the current vector $i(t)$. If the currents are sampled periodically so that the angle between two sampling instants $\Delta \varphi$ is small (Fig. 5), we can obtained in this case the instantaneous frequency of the instantaneous symmetrical component of positive sequence [7].

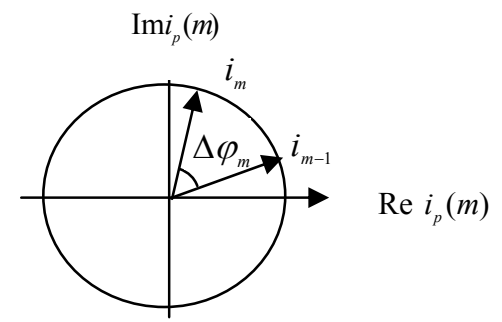

Fig. 5. Vector representation of the instantaneous vector.

$$
\begin{gathered}
\Delta \varphi \cong \sin \left(\varphi_{m}-\varphi_{m-1}\right) \\
\Delta \varphi \cong \sin \varphi_{m} \cos \varphi_{m-1}-\sin \varphi_{m-1} \cos \varphi_{m}
\end{gathered}
$$

According to the equations (11) and (12) and for a balanced three-phase system, we can write:

$$
\begin{gathered}
\operatorname{Re} i_{p}(m)=i_{m} \cos \left(\varphi_{m}\right) \\
\operatorname{Im} i_{p}(m)=i_{m} \sin \left(\varphi_{m}\right)
\end{gathered}
$$

The instantaneous symmetrical component along the axis $\alpha$ and $\beta$ are then expressed as:

$$
\begin{gathered}
i_{\alpha m}=\operatorname{Re} i_{p}(m) \\
i_{\beta m}=\operatorname{Im} i_{p}(m) \\
i_{m}=\sqrt{i_{\alpha m}^{2}+i_{\beta m}^{2}}
\end{gathered}
$$

since

$$
f_{i}=\frac{1}{2 \pi} \frac{d \varphi(t)}{d t}
$$

Yields: 


$$
\left.f_{i}=\frac{1}{2 \pi T} \frac{1}{i_{m} i_{m-1}}\left(i_{\beta m} i_{\alpha m-1}-i_{\beta m-1} i_{\alpha m}\right)\right)
$$

In practice, the current given by equations (11) and (12) are registered. The instantaneous symmetrical components long axes $\alpha$ and $\beta$ are evaluated. Hence, the instantaneous frequency is derived.

\section{Experimental Results}

The bench consists of a $5.5 \mathrm{~kW}$ three-phase squirrel cage induction motor (Fig. 6). The motor is Leroy Somer LS $132 \mathrm{~S}$, IP 55, class $\mathrm{F}, \mathrm{T}^{\circ} \mathrm{C}$ standardized $=40^{\circ} \mathrm{C}$. The nominal voltage between phases: $400 \mathrm{~V}$, the frequency of power supply $50 \mathrm{~Hz}$, nominal speed $1440 \mathrm{tr} / \mathrm{min}$, the number of the rotor slots $\mathrm{Nr}=28$. The number of stator slots $\mathrm{Ns}=48$. The stator windings are coupled in star. The motor is loaded by a powder brake. Its maximum torque $(100 \mathrm{Nm})$ is reached at the nominal speed.

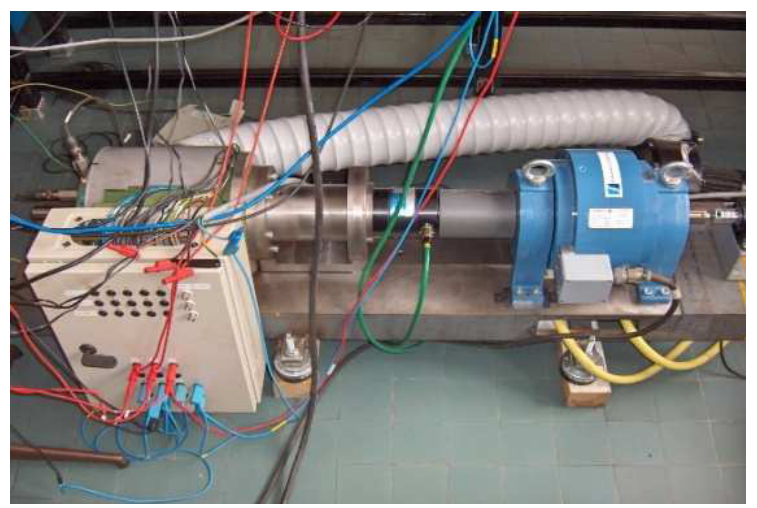

Fig. 6. Test bench.

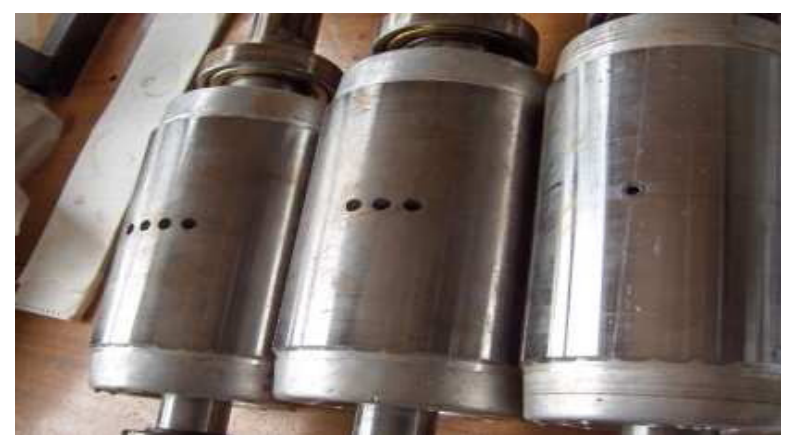

Fig. 7. Breaking of the rotor bars.

\subsection{Results of the Instantaneous Amplitude}

\subsubsection{Results of Single Phase Current}

This technique is applied to a stator current (Fig. 8) of the induction motor supplied by inverter. This machine, in this case presents a default of three broken rotor bars. Fig. 9 shows the instantaneous amplitudes of two currents one for a healthy case (dashed line) and the other for the case with fault (solid line). It shows that the instantaneous amplitude takes into account the oscillating part of period $1 / 2 \mathrm{gf}$, owner of the interesting information. In addition, the fundamental component is eliminated, making the shape of the instantaneous amplitude more readable. The spectrum of the instantaneous amplitude (Fig. 10) illustrates the representative component of the fault, namely, in this example the breaking of three rotor bars of frequency $11 \mathrm{~Hz}$.

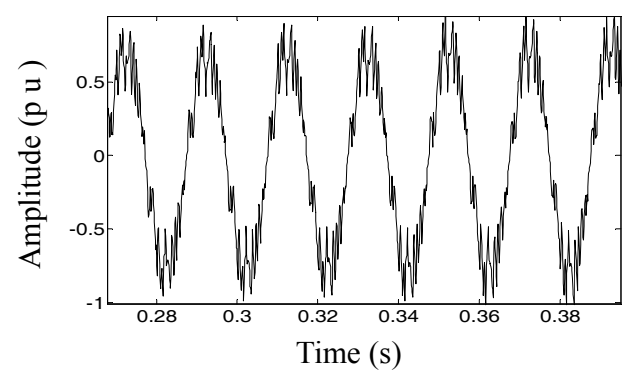

Fig. 8. Stator current with three broken rotor bars.

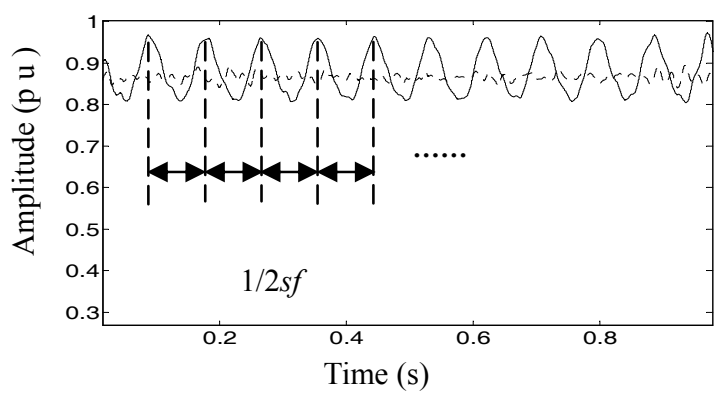

Fig. 9. Instantaneous amplitude of the stator current with three broken bars (solid line) and healthy current (dashed).

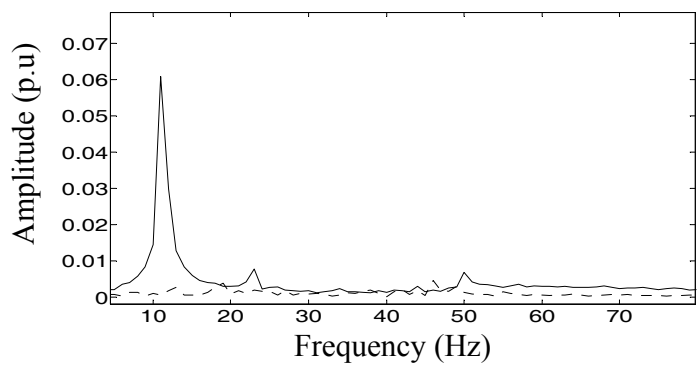

Fig. 10. Spectrum of its instantaneous amplitude. Three broken bars (solid line), healthy current (dashed).

\subsubsection{Results of Three-phase Currents System}

This technique is applied to a stator current (Fig. 11) of the induction motor supplied by inverter. This machine, in this case presents an unbalanced currents due to inter turn short-circuit of $30 \%$ stator phase.

According to Fig. 12, it may be noticed that the analogical demodulation of the current vector allows the extrac- 
tion of the negative component. The amplitude of $30 \%(-10$ $\mathrm{dB})$ of the fundamental is reached after 0,37 second. Consequently, one may conclude that this technique is more adapted to the on-line monitoring.

The numerical demodulation of the current vector is calculated for a signal sampled at $10 \mathrm{KHz}$. In order to investigate the accuracy of the opposite component (Table 1), various numbers of samples $(100,1000,10000)$ have been tested. It is found that the deviation is not due to the precision of the method used but is the result of the presence of a component lower than $50 \mathrm{~Hz}$ in the measured current. This generated an error of $3 \%$ on the fundamental magnitude. The number of 100 of samples corresponding to the ratio $f_{e} / 2 f_{s}$ represents the minimum that gives a zero mean value for all the alternative components of the stator current vector above $100 \mathrm{~Hz}$.

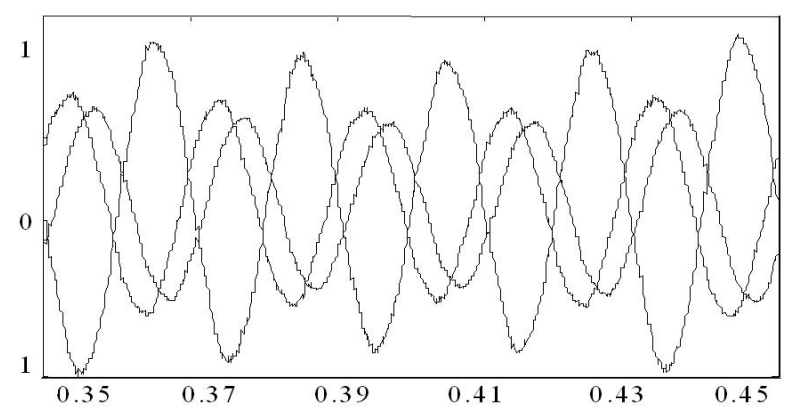

Fig. 11. Unbalanced currents due to inter turn short-circuit of $30 \%$ stator phase.

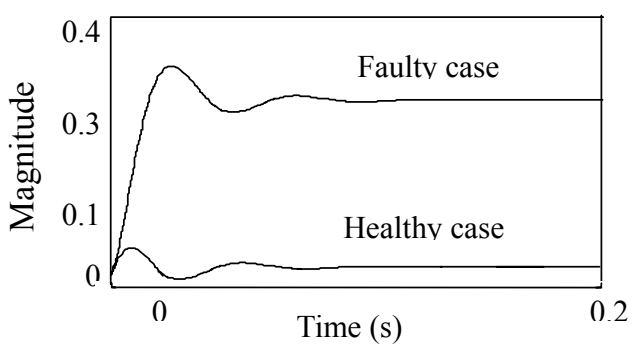

Fig. 12. Extraction of unbalance component by analogical demodulation of the stator currents vector.

Table 1. Numerical demodulation of stator currents vector

\begin{tabular}{|c|c|c|c|}
\hline Samples number & 100 & 1000 & 10000 \\
\hline Negative component magnitude & 0.33 & 0.31 & 0.30 \\
\hline Error \% & $3 \%$ & $1 \%$ & $0 \%$ \\
\hline
\end{tabular}

\subsection{Results of Instantaneous Frequency}

The instantaneous frequency is applied to the stator current of the induction motor supplied by inverter. The stator currents of the machine have been bought up in charge and during continuous operation in two cases: healthy motor and motor with broken rotor bars.

Fig. 13 shows the case of a healthy machine supplied by inverter: there is a strong deformation of the stator current that generated as a consequence a very large fluctuation of the instantaneous frequency that varies between -500 and
$500 \mathrm{~Hz}$. Consequently, we cannot characterize the operation of the healthy machine and obviously the defective one. The stator current has a very wide frequency range, which varies depending on the sampling frequency, in our case from 0 to $10,000 \mathrm{~Hz}$. All existing frequencies in the currents contribute simultaneously to the instantaneous frequency, which makes it difficult to characterize or even to extract of a relevant signature. Note that the interesting frequencies have low values for both cases: healthy or defective. The fundamental frequency $\mathrm{f}=50 \mathrm{~Hz}$ characterizes the healthy functioning, the frequency $2 \mathrm{gf}$ ( $\mathrm{g}$, slippage) characterizes the envelope of the fault of broken rotor bars. However, the initial frequency range can be reduced around the relevant frequencies in this case $160 \mathrm{~Hz}$.

Fig.14 shows the instantaneous frequency of a stator current phase of the healthy motor supplied by an inverter. The strong deformation of the stator current (red line) was attenuated by the reduction of the frequency range up to $160 \mathrm{~Hz}$ (black line) and which generated as a consequence attenuation in the instantaneous frequency fluctuation. Note that despite the reduced modulation around $50 \mathrm{~Hz}$, the instantaneous frequency of the phase current may, however, characterize a healthy functioning of the machine.

Fig. 15 shows the instantaneous frequency of a stator current phase in the case of an inverter supply of the machine with four broken bars. It is noted that the modulation of the stator current amplitude appears also as a form of equidistant envelopes of period $1 / 2 g f$ of 0.3 second and a maximum frequency of $52 \mathrm{~Hz}$.
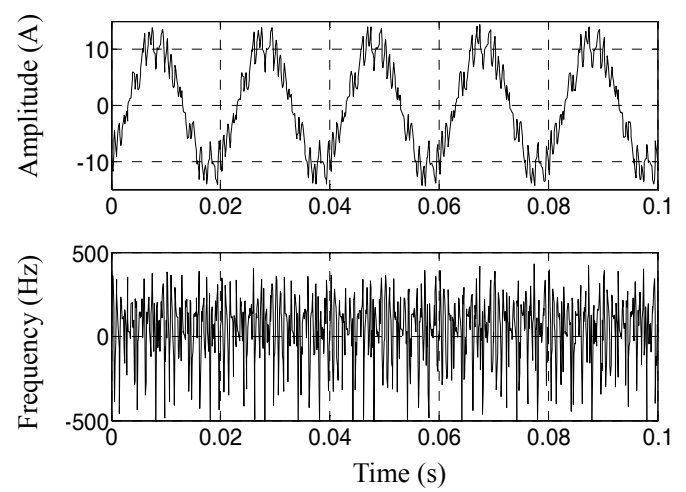

Fig. 13. Phase current and instantaneous frequency of a healthy motor supplied by an inverter.
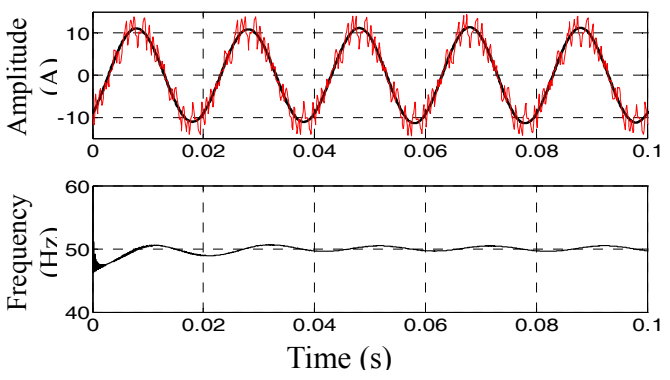

Fig. 14. Phase current and instantaneous frequency of a healthy motor supplied by inverter. 


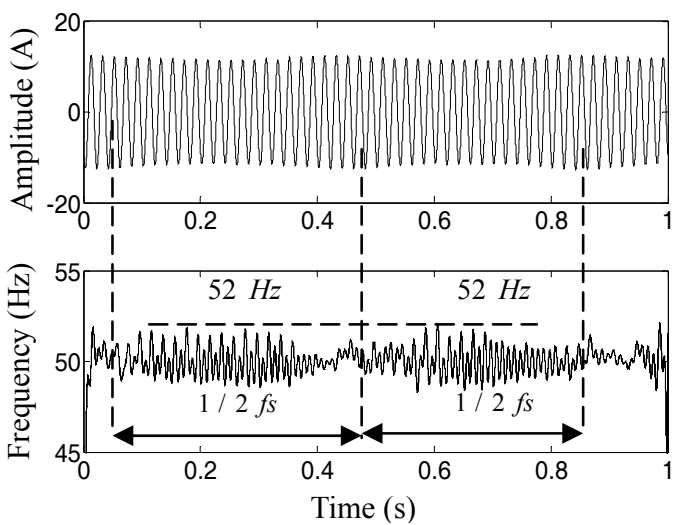

Fig. 15. Instantaneous frequency of a motor phase supplied with an inverter with 04 broken bars.

\section{Conclusion}

The stator current represents a data recording medium very rich in information. The traditional spectral analysis of the fault does not allow better exploitation of the current signal because of the proximity of the characteristic components of the fault, some Hertz, to the dominant fundamental component at $50 \mathrm{~Hz}$. The representation of one part of the signal: the instantaneous frequency or the instantaneous amplitude makes it possible to withdraw the fundamental one and maintain only the relevant information, in fact, the instantaneous amplitude and frequency. These have the advantage of being applied for an on-line monitoring and a diagnosis without being masked by the fundamental of the stator current.

\section{References}

[1] G.B. Kilman and J. Stein. Methods of motor current signature analysis. Electr. Mach. \& Power Syst, Vol.5, No. 1, pp.41-52, 1999.

[2] A.J.M. Cardoso, S.M.A. Cruz, J.F.S. Carvalho, and E.S. Saraiva. Rotor cage fault diagnosis in induction motors by park's vector approach. IEEE, IAS'95 Orlando, Florida, Oct., pp642-646, 1995.

[3] Lebaroud Abdesselam, Clerc Guy, "Classification of Induction Machine Faults by Optimal Time-Frequency Representations," Industrial Electronics, IEEE Transactions, pp.4290- 4298, Vol.55, No.12, Dec., 2008.

[4] Thomson W. T., M Fenger "Current signature analysis to detect inductions motor faults "Introduction" IEEE Industry Applications Magazine_July/August 2001.

[5] Lebaroud Abdesselam, Abdelmalek Khezzar, Ammar Bentounsi, Guy Clerc "Comparison of the Induction Motors Stator Fault Monitoring Methods Based on Current Negative Symmetrical Component," European power electronics and drives, EPE Journal, Vol. 17, No.1, Mar., 2007.
[6] Francesco Cupertino et all, “Analysis Techniques for Detection of IM Broken Rotor Bars After Supply Disconnection," IEEE transactions on industry applications, Vol.40, No.2, 2004.

[7] Lebaroud Abdesselam, Clerc Guy "Diagnosis of Induction Motor Faults Using Instantaneous Frequency Signature Analysis, International conference in electrical machines (ICEM’08), Portugal, Sep., 2008.

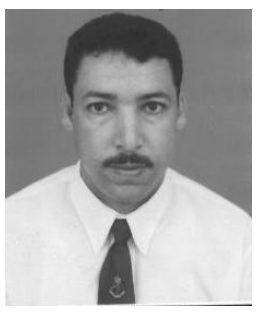

Medoued Ammar was born in 1971, in Skikda, Algeria. He received the Engineer Diploma and MSc Degree, in 1994 and 1998, respectively, from University of Skikda, Algeria in Electrical Engineering. He is currently an Lecturer at the University of Skikda and the Head of Electrical Engineering Department, Algeria. His main research field is Electrical Machine Diagnosis.

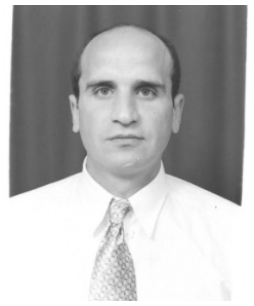

Abdesselam Lebaroud was born in Constantine, Algeria, in 1969, He received the $\mathrm{PhD}$ degree in electrical engineering from University Claude Bernard Lyon I, Ampere laboratory, France, in 2007. Currently, he is a Associate Professor at the Electrical Engineering Institute of Skikda University. He carried out researches on diagnosis of electrical machines at LEG of Constantine.

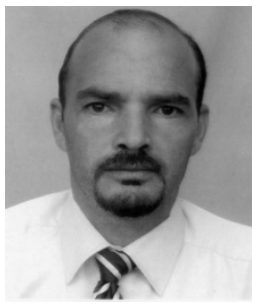

Boukadoum Ahcene was born in 1969, in Skikda, Algeria. He received the Engineer Diploma and PhD Degree in Electromechanics, in 1996 and 2000, respectively, from State University of Technology of Tachkent Beirnouni, Ouzbékistan, in Energy Systems. He is currently an Associate Professor at the University of Skikda and the Director of LES Laboratory, Algeria. His main research field is Electrical Machine Diagnosis.

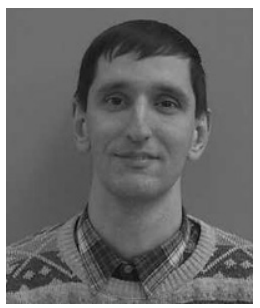

Guy Clerc was born in Libourne, France, on November 30, 1960. He received the Enginer's degree and the $\mathrm{PhD}$ in electrical engineering from the Ecole Centrale de Lyon, France, in 1984 and 1989, respectively. He is Professor of Universities. He teaches electrical engineering at the "University Claude Bernard Lyon I" in France. He carried out researches on control and diagnosis of induction machines 